\title{
Multiple Object Localization in Underwater Wireless Communication Systems using the Theory of Gravitation
}

\author{
Ruhul Amin Khalil ${ }^{\dagger}$, Nasir Saeed ${ }^{\diamond}$, Tariqullah Jan ${ }^{\dagger}$, and Majid Ashraf ${ }^{\dagger}$ \\ $\dagger$ Department of Electrical Engineering \\ University of Engineering and Technology Peshawar \\ Email: [1.ruhulamin, 3. tariqullahjan and 4. majid] @uetpeshawar.edu.pk \\ $\diamond$ King Abdullah University of Science and Technology \\ Thuwal, Makkah, Kingdom of Saudi Arabia \\ Email: mr.nasir.saeedeieee.org
}

\begin{abstract}
Localization of underwater objects has always been a challenging task due to the unguided and misty nature of the underwater environment. Therefore, in this paper, a novel algorithm has been proposed to find the correct location of various underwater objects in real-time underwater communication networks. The proposed algorithm is based on collective utilization of gravity field vector and gravity gradient tensor signals for object localization. It computes the localization of numerous underwater objects in a non-linear scenario by utilizing modified Levenberg-Marquardt algorithm. The study uses singleobject localization scenario to carry out estimation of the initial coordinates and mass calculation of the underwater objects. In order to validate the proposed algorithm, two objects based artificial navigation model has been used for testing purpose. The results obtained were precise and accurate, hence, the proposed method is a good strategy to localize multiple underwater objects.
\end{abstract}

Index Terms-Underwater objects, Localization, gravity field vector, Levenberg-Marquardt

\section{INTRODUCTION}

With the advancements in wireless communication systems, high-speed data transmission is pervasive in modern sciences. These robust communication systems work efficiently in the air but, when it comes to underwater applications, their performance degrades. The reasons behind this performance degradation, are the un-guided and hazy nature of the underwater environment. These underwater wireless communication systems utilize various data communication carriers such as radio-frequency (RF) waves, acoustic waves, and optical waves [1]. With air as a transmission medium, the RF communication system provides higher data rates of transmission over long distances utilizing very moderate power. However, such effective communication of RF cannot be achieved in an underwater environment due to its high tendency to absorb electromagnetic signals. Acoustics systems deliver long-range communications, but its data rates are much low due to the low sound speed in water [2]. On the other hand, optical wireless communication provides higher data rate and cost-efficient system design [3]. Comparison of various underwater communications systems is depicted in Fig .1. Apart for the ranging capability and data rate, it is much important that which one of these systems are efficient to locate underwater objects knowing the misty nature of the underwater environment.

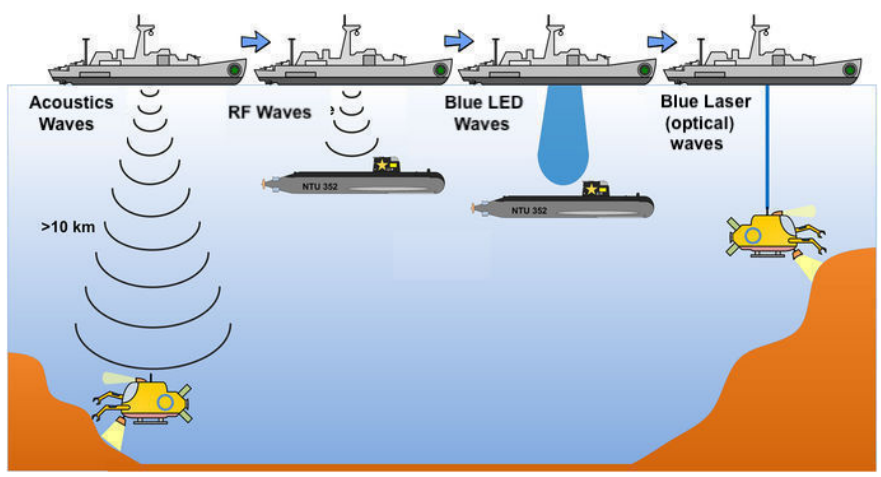

Fig. 1. Comparison of various underwater communications systems.

Localization of objects in underwater environments has attained considerable reputation in both military and civil applications. Many traditional techniques such as Global Positioning system (GPS), Autonomous Underwater Vehicles (AUVs), Ultra-wide band (UWB), Dive and Rise (DNR), and Wider positioning system (WPS) have been used to estimate the accurate position of objects [4]-[6]. Due to the developments in technology and security measurements, these aviation and underwater objects can be made invisible to these traditional techniques for finding locations. In contrast, other techniques such as gravity signals can be utilized to detect and find the location of these invisible objects using either acoustically or magnetically invisible objects.

Techniques known as gravity field vectors (GFV) and gravity gradient tensor (GGT) have been recently developed, to locate and monitor movements of various objects in aerial and underwater environments. Gravitational potential of earth and 
related parameters such as centripetal force are due to the sun, planets, moon and other natural forces. The analysis of variations in the acceleration due to such gravitational potential is known as gravity gradiometry and its measuring gadgets are known as gravity gradiometers. These gravity gradiometers are significantly used due to its instinctive component along z-direction that is basically the rate of change of gravity in vertical direction with a given height. It can be measured by calculating the gravitational pull at two different positions by a small vertical distance and dividing by their separation distance. The gravity gradient (GG) is the rate of change of acceleration due to gravity, however, gravity gradient tensor (GGT) is basically the degree of rate of change of gravity field vector (GFV) in all the three dimensions perpendicularly. The system deployment of these techniques is cost efficient as compared to the traditional techniques available in the literature. In [7], the detailed structural design and implementation of the base stations for localization of various objects have been presented. An analytical formula has been developed recently using GGTs for location detection of objects in air [8]. This formula was developed by combining the GFV and GG signals for object localization. The localization of objects here was measured by using a meter known as gravity gradiometer and found to be approximately equal to $10^{-4} \mathrm{E}$.

Recently, localization of single underwater object by using GF and horizontal GGT signals is proposed in [9]. Therefore, this paper proposes an analytical solution for the localization of multiple objects in an underwater environment by using GF and GGT. To localize various objects simultaneously in a real-time scenario, two algorithms known as GFV and GGT signals have been utilized. Then computations are carried out to calculate the unknown masses and location coordinates of these multiple objects. The modified Levenberg-Marquardt algorithm has been utilized for these unknown findings and afterward, these computations are regularized in a non-linear scenario. Compared to the standard Levenberg-Marquardt, the proposed algorithm is more robust and offers increased stability. Initially, single object localization problem has been utilized to determine the location of an object automatically and find its approximate coordinates. Later on, a model has been presented to localize two objects in the underwater environment in a much accurate way.

Rest of the paper is organized as follows: In Section II, the focus has been laid on single object localization in an underwater environment using a 3-D coordinate system. In Section III, critical analysis has been carried out for multiple objects localization in the same underwater environment. Section IV includes simulations results obtained in the considered scenario and discussion made. In Section V, system evaluation is discussed followed by the conclusions.

\section{UNDERWATER Single ObJECT LOCALIZATION}

This paper considers a scenario which consists of $n$ base stations and a single underwater target object. Also, a 3dimensional rectangular coordinate system is commenced, in which the horizontal surface consists of the $x-y$ coordinates and the $z$-coordinate lie vertically downward as shown in Fig. 2 . The 3 -D coordinate mass $P(x, y, z)$ causes some gravity potential $\delta^{k}$ at the $k$ th base station . This gravity potential $\delta^{k}$ is produced with a constant density $\rho$ with negligible inertial centrifugal force as follows [10]-[12]

$$
\delta^{k}=\iiint_{0} \frac{G_{\rho}}{D^{k}} d x d y d z,
$$

where $D^{k}=\left(\left(\Delta x^{k}\right)^{2}+\left(\Delta y^{k}\right)^{2}+\left(\Delta z^{k}\right)^{2}\right)^{\frac{1}{2}}$ provides the separation between the coordinates of body mass $O(x, y, z)$ and the base station $P_{k}\left(u^{k}, v^{k}, w^{k}\right), \Delta x^{k}=x-u^{k}, \Delta y^{k}=$ $y-v^{k}, \Delta z^{k}=z-w^{k}$, and $G=6.67 \times 10^{-11} \mathrm{~kg}^{-1} \mathrm{~m}^{3} \mathrm{~s}^{-2}$ is the universal gravitational constant. Also, the $\rho$ is with small centrifugal force so $G_{\rho}$ becomes approximately equal to $G$.

For the $k$ th base station, the gravitational field vector $f^{k}$ is expressed as

$$
f^{k}=\nabla \delta^{k}
$$

and the symmetry of gravity gradient tensor $S^{k}$ is given as

$$
S^{k}=\nabla \nabla \delta^{k}\left[\begin{array}{ccc}
S_{x x}^{k} & S_{x y}^{k} & S_{x z}^{k} \\
S_{y x}^{k} & S_{y y}^{k} & S_{y z}^{k} \\
S_{z x}^{k} & S_{z y}^{k} & S_{z z}^{k}
\end{array}\right]
$$

As, the GGTs calculate the degree of rate of change of GFV in the $x-, y-$ and $z-$ directions. So, the coordinate mass can be estimated well enough by a single mass point, if its separation from the site of observation is at least three or more times larger as compared to the target dimensions [13]. After such a consideration, (2) can be approximated as

$$
f^{k}=\left\{\begin{array}{ccc}
f_{x}^{k} & f_{y}^{k} & f_{z}^{k}
\end{array}\right\}=\frac{G M}{\left(D^{k}\right)^{3}}\left\{\Delta x^{k} \Delta y^{k} \Delta z^{k}\right\},
$$

where $M$ represents the mass of the underwater object. According to [8] [14], (3) can be approximated as

$$
S^{k}=\frac{-G M}{\left(D^{k}\right)^{5}}\left[\begin{array}{ccc}
\left(D^{k}\right)^{2}-3\left(\Delta x^{k}\right)^{2} & -3 \Delta x^{k} \Delta y^{k} & -3 \Delta x^{k} \Delta z^{k} \\
-3 \Delta y^{k} \Delta x^{k} & \left(D^{k}\right)^{2}-3\left(\Delta y^{k}\right)^{2} & -3 \Delta y^{k} \Delta z^{k} \\
-3 \Delta z^{k} \Delta x^{k} & -3 \Delta z^{k} \Delta y^{k} & \left(D^{k}\right)^{2}-3\left(\Delta z^{k}\right)^{2}
\end{array}\right] .
$$

With utilization of equation (4) and (5) at the $k$ th base station, an analytic formula of the barycenter location $\left(x_{r}^{k}, y_{r}^{k}, z_{r}^{k}\right)$ and mass $M_{r}^{k}$ of a single object lying in an underwater environment.

$$
\left\{\begin{array}{c}
{\left[x_{r}^{k} y_{r}^{k} z_{r}^{k}\right]=\left[u^{k} v^{k} w^{k}\right]-\frac{2\left[f_{x}^{k} f_{y}^{k} f_{z}^{k}\right]}{S_{x x}^{k}+S_{y y}^{k}-\sqrt{\left(S_{x x}^{k}+S_{y y}^{k}\right)^{2}+4 S_{x y}^{k 2}}}} \\
M_{r}^{k}=\frac{4\left(f_{x}^{k 2}+f_{y}^{k 2}+f_{z}^{k 2}\right)^{\frac{3}{2}}}{G\left[S_{x x}^{k}+S_{y y}^{k}-\sqrt{\left(S_{x x}^{k}+S_{y y}^{k}\right)^{2}+4 S_{x y}^{k 2}}\right]^{2}}
\end{array}\right.
$$

where as the subscript $r$ resembles to the localization of single underwater object. Using this localization technique, if the single object lying in underwater environment is approximated well enough as a point mass. Then utilizing (6), the barycenter locations and masses can be obtained in similar manner for all the $n$ number of gravitational base stations. 


\section{UnderWATER MUlTiPle OBJECTS LOCALIZATION}

\section{A. Principle for Localization of Multiple objects}

Localization of multiple underwater objects by gradiometer base stations is depicted in Fig. 2, where there are $n$ number of underwater base stations and $m$ objects where $m \leq n$.

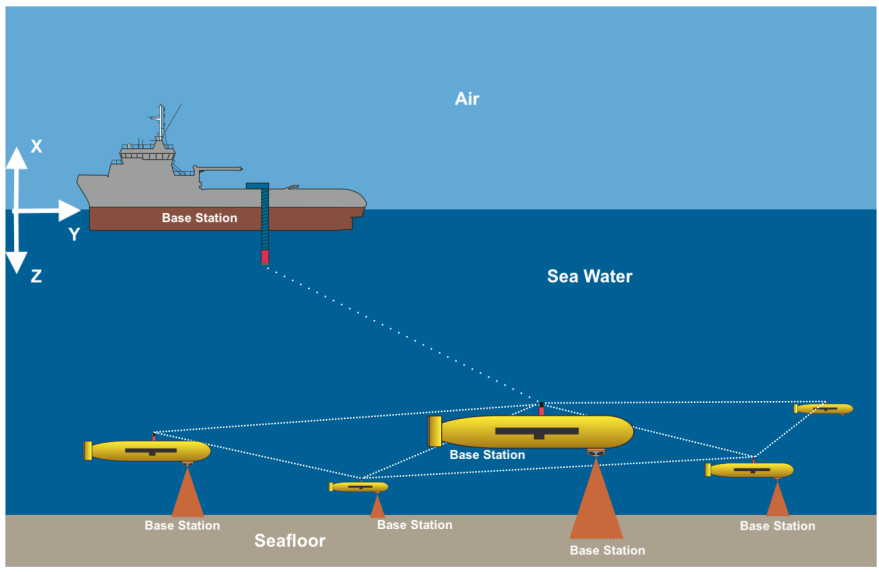

Fig. 2. Localization of multiple underwater objects by gradiometer base stations.

By using (4) and (5) at the $k$ th base station, the parameters $f^{k}$ and $S^{k}$ are estimated as follows

$$
\begin{gathered}
f^{k}=\left\{f_{x}^{k} f_{y}^{k} f_{z}^{k}\right\}=\sum_{l=1}^{m} G \frac{M^{k}}{\left(D^{k l}\right)^{3}}\left\{\Delta x^{k l} \Delta y^{k l} \Delta z^{k l}\right\}, \\
S^{k}=\sum_{l=1}^{m} \frac{-G M^{l}}{\left(D^{k l}\right)^{5}}\left[\begin{array}{ccc}
\left(D^{k l}\right)^{2}-3\left(\Delta x^{k l}\right)^{2} & -3 \Delta x^{k l} \Delta y^{k l} \\
-3 \Delta y^{k l} \Delta x^{k l} & \left.\left(D^{k l}\right)^{2}-3 \Delta y^{k l}\right)^{2} & -3 \Delta x^{k l} \Delta z^{k l} \\
-3 \Delta z^{k l} \Delta x^{k l} & -3 \Delta z^{k l} \Delta y^{k l} & \left(D^{k l}\right)^{2}-3\left(\Delta z^{k l}\right)^{2}
\end{array}\right] .
\end{gathered}
$$

In (8), separation between the $k$-th base station $P_{k}\left(u^{k}, v^{k}, w^{k}\right), \Delta x^{k l}=x^{l}-u^{k}, \Delta y^{k l}=y^{l}-v^{k}$, $\Delta z^{k l}=z^{l}-w^{k}$, and the mass of $l$ th body $O_{l}\left(x^{l}, y^{l}, z^{l}\right)$ is represented by $D^{k l}=\left[\left(\Delta x^{k l}\right)^{2}+\left(\Delta y^{k l}\right)^{2}+\left(\Delta z^{k l}\right)^{2}\right]^{1 / 2}$. Also, the $l$ th body mass is expressed by $M^{l}$.

From (6), the parameters $S_{x z}^{k}, S_{x x}^{k}, S_{z z}^{k}$, and $f_{z}^{k}$ can be re-written as

$$
\left\{\begin{array}{c}
S_{x z}^{k}=\sum_{l=1}^{m} S_{x z}^{k l}=\mathbf{U}_{(1 \times m)}^{k} \mathbf{S}_{x z(m \times 1)}^{k} \\
S_{x x}^{k}=\sum_{l=1}^{m} S_{x x}^{k l}=\mathbf{V}_{(1 \times m)}^{k} \mathbf{S}_{x z(m \times 1)}^{k} \\
S_{z z}^{k}=\sum_{l=1}^{m} S_{z z}^{k l}=\mathbf{W}_{(1 \times m)}^{k} \mathbf{S}_{x z(m \times 1)}^{k} \\
f_{z}^{k}=\sum_{l=1}^{m} f_{z}^{k l}=\mathbf{X}_{(1 \times m)}^{k} \mathbf{S}_{x z(m \times 1)}^{k}
\end{array},\right.
$$

where $\mathbf{S}_{x z}^{k}=\left[S_{x z}^{k 1} \cdots S_{x z}^{k m}\right]_{(m \times 1)}^{T}, T$ in the superscript indicates the transpose operator. The vector $\mathbf{S}_{x z(m \times 1)}^{k}$ relates each part to the $x-y$ coordinates of the GGTs that is produced by the mass body. Hence, all the elements present in $\mathbf{S}_{x z(m \times 1)}^{k}$ can be collectively determined at the $k$-th base station. In contrast, these elements cannot be determined on individual basis. $\mathbf{S}_{x z(m \times 1)}^{k}$ in (9) signify some undetermined intermediate state variables. However, the parameters $S_{x z}^{k}, S_{x x}^{k}, S_{z z}^{k}$ and $f_{z}^{k}$ are measurable. On the right hand side of (9), the matrices
$\mathbf{U}^{k}, \mathbf{V}^{k}, \mathbf{W}^{k}, \mathbf{X}^{k}$ only possess the geometrical association among the considered base stations and the mass bodies. These matrices are defined as

$$
\left\{\begin{array}{rl}
\mathbf{U}_{(1 \times m)}^{k} & =\left[U^{k 1} \cdots U^{k m}\right]_{(k \times m)} \\
\mathbf{V}_{(1 \times m)}^{k} & =\left[V^{k 1} \cdots V^{k m}\right]_{(k \times m)} \\
\mathbf{W}_{(1 \times m)}^{k} & =\left[W^{k 1} \cdots W^{k m}\right]_{(k \times m)} \\
\mathbf{X}_{(1 \times m)}^{k} & =\left[X^{k 1} \cdots X^{k m}\right]_{(k \times m)}
\end{array},\right.
$$

and

$$
\left\{\begin{array}{c}
U^{k l}=1 \\
V^{k l}=\frac{\left[\left(D^{k l}\right)^{2}-3\left(\Delta x^{k l}\right)^{2}\right]}{\left.-3 \Delta x^{k l} \Delta z^{k l}\right]} \\
W^{k l}=\frac{\left[\left(D^{k l}\right)^{2}-3\left(\Delta z^{k l}\right)^{2}\right]}{-3 \Delta x^{k l} \Delta z^{k l}} \\
X^{k l}=\frac{\left(D^{k l}\right)^{2}}{3\left(\Delta x^{k l}\right)^{2}}
\end{array}\right.
$$

respectively. By using (8) and (9), the relationship among the three parameters, $S_{x y}^{k}$ and $S_{y z}^{k}$ with $S_{x z}^{k l}$ is given by

$$
\begin{aligned}
& S_{x y}^{k}=\sum_{l=1}^{m}\left(\frac{\Delta y^{k l}}{\Delta z^{k l}}\right) S_{x z}^{k l} \\
& S_{y z}^{k}=\sum_{l=1}^{m}\left(\frac{\Delta y^{k l}}{\Delta x^{k l}}\right) S_{x z}^{k l}
\end{aligned}
$$

Consider that

$$
\mathbf{H}^{k}=\left[\begin{array}{c}
\Delta y^{k 1} / \Delta z^{k 1} \cdots \Delta y^{k m} / \Delta z^{k m} \\
\Delta y^{k 1} / \Delta z^{k 1} \cdots \Delta y^{k m} / \Delta z^{k m}
\end{array}\right] .
$$

Therefore, utilizing the above consideration, $\mathbf{J}^{k}=$ $\left[S_{x y}^{k} S_{y z}^{k}\right]^{T}$, (12) and (13) can be transformed into a matrix form as

$$
\mathbf{H}^{k} \mathbf{S}_{x z(m \times 1)}^{k}=\mathbf{J}^{k}
$$

In order to calculate the undefined variables, $\mathbf{S}_{x z}^{k}$ can be represented in the form a least-square estimation problem, i.e., [15]

$$
\mathbf{S}_{x z(m \times 1)}^{k}=\left[\left(\mathbf{H}^{k}\right)^{T} \mathbf{H}^{k}\right]^{-1}\left(\mathbf{H}^{k}\right)^{T} \mathbf{J}^{k}
$$

It is not always possible to find the inverse of matrix $\left(\mathbf{H}^{k}\right)^{T}$ $\mathbf{H}^{i}$, therefore, Tikhonov regularization [16] can be utilized, i.e.,

$$
\mathbf{S}_{x z(m \times 1)}^{k}=\left[\left(\mathbf{H}^{k}\right)^{T} \mathbf{H}^{k}+\mu \mathbf{I}\right]^{-1}\left(\mathbf{H}^{k}\right)^{T} \mathbf{J}^{k}
$$

where $\mu>0$ is a regularization parameter.

In the next step, (10) is multiplied by (16) on both sides as follows

$$
\left\{\begin{aligned}
\mathbf{U}_{(1 \times m)}^{k} \mathbf{H}_{L}^{k} \mathbf{J}^{k} & =\mathbf{U}_{(1 \times m)}^{k} \mathbf{S}_{x z(m \times 1)}^{k} \\
\mathbf{V}_{(1 \times m)}^{k} \mathbf{H}_{L}^{k} \mathbf{J}^{k} & =\mathbf{V}_{(1 \times m)}^{k} \mathbf{S}_{x z(m \times 1)}^{k} \\
\mathbf{W}_{(1 \times m)}^{k} \mathbf{H}_{L}^{k} \mathbf{J}^{k} & =\mathbf{W}_{(1 \times m)}^{k} \mathbf{S}_{x z(m \times 1)}^{k} \\
\mathbf{X}_{(1 \times m)}^{k} \mathbf{H}_{L}^{k} \mathbf{J}^{k} & =\mathbf{X}_{(1 \times m)}^{k} \mathbf{S}_{x z(m \times 1)}^{k}
\end{aligned}\right.
$$

where $\mathbf{H}_{L}^{k}=\left[\left(\mathbf{H}^{k}\right)^{T} \mathbf{H}^{k}+\mu \mathbf{I}\right]^{-1}\left(\mathbf{H}^{k}\right)^{T}$. Now, using equation (9) and equation (17) yields 


$$
\left\{\begin{array}{rl}
\mathbf{U}_{(1 \times m)}^{k} \mathbf{H}_{L}^{k} \mathbf{J}^{k} & =S_{x z}^{k} \\
\mathbf{V}_{(1 \times m)}^{k} \mathbf{H}_{L}^{k} \mathbf{J}^{k} & =S_{x x}^{k} \\
\mathbf{W}_{(1 \times m)}^{k} \mathbf{H}_{L}^{k} \mathbf{J}^{k} & =S_{z z}^{k} \\
\mathbf{X}_{(1 \times m)}^{k} \mathbf{H}_{L}^{k} \mathbf{J}^{k} & =f_{z}^{k}
\end{array} .\right.
$$

By analyzing (18), it is realized that intermediate variables $\mathbf{S}_{x z}^{k}$ have been completely eliminated. Its left-side not only involves the geometrical association among the selected base stations and mass bodies, but also, the quantity $\mathbf{J}^{k}$ at each base station. Its right-side is comprised of deliberate signals parameters $S_{x z}^{k}, S_{x x}^{k}, S_{z z}^{k}$ and $f_{z}^{k}$. This equation is having another distinctive feature known as distribution of mass for the considered mass bodies that is permanently removed. This in turn reduces the number of known quantities but although stabilizes the overall scenario. The reason behind is that all the unknown quantities in the equation are measuring coordinates having same unites and dimensionality.

Considering $m$ underwater objects and $n$ gravity base stations, the non-linear scenario where $(m \leq n)$ in (18) is re-expressed as

$$
\left[\begin{array}{c}
\mathbf{U}_{(1 \times m)}^{1} \mathbf{H}_{L}^{1} \mathbf{J}^{1} \cdots \mathbf{U}_{(1 \times m)}^{n} \mathbf{H}_{L}^{n} \mathbf{J}^{n} \\
\mathbf{V}_{(1 \times m)}^{1} \mathbf{H}_{L}^{1} \mathbf{J}^{1} \cdots \mathbf{V}_{(1 \times m)}^{n} \mathbf{H}_{L}^{n} \mathbf{J}^{n} \\
\mathbf{W}_{(1 \times m)}^{1} \mathbf{H}_{L}^{1} \mathbf{J}^{1} \cdots \mathbf{W}_{(1 \times m)}^{n} \mathbf{H}_{L}^{n} \mathbf{J}^{n} \\
\mathbf{X}_{(1 \times m)}^{1} \mathbf{H}_{L}^{1} \mathbf{J}^{1} \cdots \mathbf{X}_{(1 \times m)}^{n} \mathbf{H}_{L}^{n} \mathbf{J}^{n}
\end{array}\right]^{\prime}=\left[\begin{array}{c}
S_{x z}^{1} \cdots S_{x z}^{n} \\
S_{x x}^{1} \cdots S_{x x}^{n} \\
S_{z z}^{1} \cdots S_{z z}^{n} \\
f_{z}^{1} \cdots f_{z}^{n}
\end{array}\right]^{\prime}
$$

In (19), non-linear equations have been obtained for the barycenter coordinates that represent mass bodies. According to [17], nonlinear solutions such as the modified LevenbergMarquardt algorithm can be easily applied to solve such a scenario.

It is known that, the initial barycenter coordinates of the considered $m$ underwater objects play an important role in solution of such problems. It provides the possibility to utilize the predicted barycenter coordinates for localization of single object algorithm that is already discussed in Section-II. Here $n$ number of barycenter coordinates can easily be predicted and calculated using the signals received from $n$ base stations. The $n$ barycenter coordinates that behaves as initialized values in (19), one can select any of the barycenter coordinates based on the criterion $m(m \leq n)$. Increase in the separation between base stations and mass bodies results in rapid decrease in amplitude of GGTs and GF at that base station. So, it can predict by taking help from the nearby object. Therefore, mostly it is considered that these predicted barycenter coordinates are reasonable choice for the localization. Also, the already measured positions of objects can be considered as starting positions for the measurement of next reading. Finally, to attain the masses of the mass bodies, the probable localities needs to be substituted in (7) and (8).

\section{B. Approximation of Regularization Parameters}

It has always been challenging to choose a proper parameter $\mu$ for regularization process [18]. The parameter $\mu$ is preferred to be chosen by the method of cross-validation [19]. In the cross-validation technique, the experimental data is first distributed into a training set and later on into testing set. The parameter $\mu$ is distributed over a range of $\left(0, \mu_{\max }\right)$. After range selection, some possible and defined values $M_{\mu}$ are taken equally on a logarithmic scale. The training set having a non-linear problem in equation (19) is utilized to find out all possible values of $\mu$. The solutions of non-linear problems in testing set provide help in the computation of the GGTs and gravity field signals at any desired base station. These calculated signals are compared with the testing data set, and optimal regularization parameter has been chosen based on the minimum difference. The regularization parameters are usually acknowledged as the ultimate coordinates for finding the location and define the respective masses of these underwater objects.

\section{RESULTS AND DisCUSSION}

\section{A. Simulation Environment}

Matlab is used for the all the simulation results whereas the superconducting gravity meter with the sensitivity level of $1 n \mathrm{Gal}$ is conisdered to compute the gravity [20], [21].

According to this setup, the noise level is set to $0.05 \mathrm{E}$ for GGT and $0.05 \mu$ Gal for GF. For validation and feasibility test of the presented algorithm, a navigation model has been developed for two underwater objects (object $\mathrm{R}$ and object $\mathrm{S}$ ). Object $\mathrm{R}$ is cubic in shape with dimensions $12 m \times 12 m \times 12 m$, and its body mass is equal to $4.32 \times 10^{6} \mathrm{~kg}$. However, object $\mathrm{S}$ is prismatic in shape with dimensions $24 \mathrm{~m} \times 8 \mathrm{~m} \times 8 \mathrm{~m}$ and body mass of $4.61 \times 10^{6} \mathrm{~kg}$.

The exact trajectory followed by object $\mathrm{R}$ is having the following coordinates

$$
\begin{gathered}
x=115.75-(k-1) \times \Delta t \times 2315 \\
y=9.375 \times 10^{-4} \times x^{2}+30 \\
z=10
\end{gathered}
$$

where $k=(1,2, \cdots, 21)$. The exact trajectory followed by object $\mathrm{S}$ is having the following coordinates

$$
\begin{gathered}
x=115.75-(k-1) \times \Delta t \times 2315 \\
y=0.75 \times x \\
z=50
\end{gathered}
$$

given by solid blue line as shown in Fig. 3. The moving speed of both the objects $\mathrm{R}$ and $\mathrm{S}$ is same and taken as 2.315 $\mathrm{km} / \mathrm{h}$ with different starting positions as $(115.75 \mathrm{~m}, 42.56 \mathrm{~m}$, and $10 \mathrm{~m})$ and $(115.75 \mathrm{~m}, 86.81 \mathrm{~m}$, and $50 \mathrm{~m})$ respectively. As depicted from Fig.3, the base stations are installed at four distinctive positions. With defined GV and GGTs, these base stations are considered to be located at seawater surface where $z=0 m$.

For simulation purpose, 21 number of observations were recorded with equal integral part of $t=7.5$ seconds. For scenario 1, the coordinates of the two base stations coordinates for first scenario are $(50 \mathrm{~m}, 90 \mathrm{~m}$, and $0 \mathrm{~m})$ and $(50 \mathrm{~m}, 10 \mathrm{~m}$, and $0 \mathrm{~m}$ ). Similarly, the coordinates for the two base stations for scenario 2 are $(100 m, 100 m$, and $0 m)$ and $(60 m, 10 m$, 


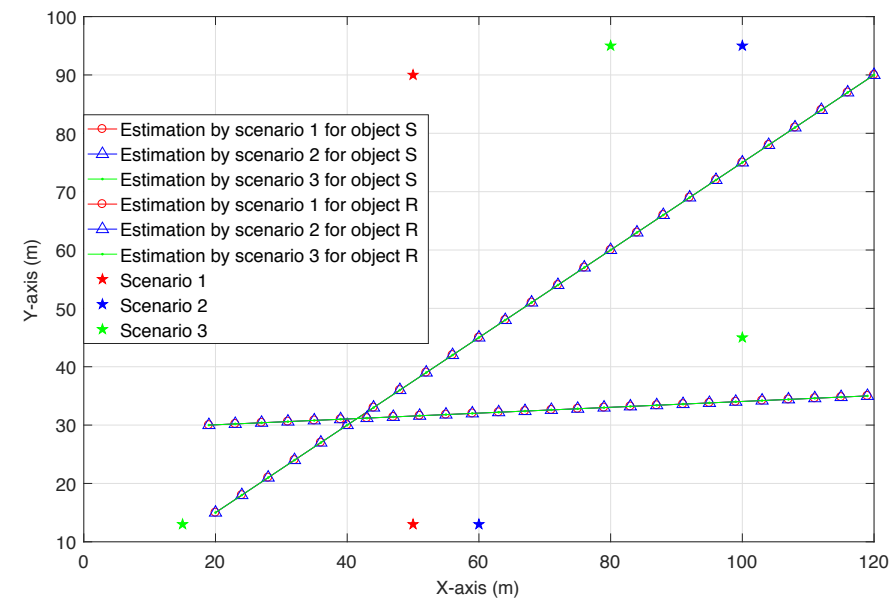

Fig. 3. Tracking of multiple underwater objects.

and $0 \mathrm{~m})$. Also, both of these scenarios are $(80 \mathrm{~m}, 100 \mathrm{~m}$, and $0 \mathrm{~m}),(30 \mathrm{~m}, 10 \mathrm{~m}$, and $0 \mathrm{~m})$, for the third scenario $(100 \mathrm{~m}, 50 \mathrm{~m}$, and $0 \mathrm{~m}$ ). Due to the small scale recorded data, it is considered to be serving as both the training and testing sets. During the experiments conduction, the parameter for regularization $\mu$ is taken to be in range of $10^{-8}-10^{-3}$. The complete number of parameters for regularization that are used during the testing phase was 16 .

\section{B. Experimental Results and Discussion}

As resulted after the simulations, the trajectory followed by both the objects $\mathrm{R}$ and $\mathrm{S}$ is depicted in Fig. 4. The trajectory followed by both objects is very nearer to the actual trajectory followed by the objects thus agreeing with the parameters considered in all three scenarios. The deviance of the estimated location positions from the original ones are less than $0.2 m$ in all directions. Considering this deviance and size of both the objects, their positions can be estimated to be lying inside the objects R and S. Furthermore, using the same algorithm, these results becomes more accurate if the distance of the underwater objects from the base station increases.

During the next phase of experimentation, four possible initial positions were assumed that comes from barycenter coordinates. Now, with the algorithm of multiple underwater objects localization, two best initial positions of the two objects are selected from the four possible positions. This selection results more than $90 \%$ accuracy and provides the true localization of objects from their initial locations.

Noisy environment has been considered, as the models for actual noise are currently not available. One best noise consideration is to utilize the random noise model [22] for the simulation purpose in the current experimental setup. In scenario 1 , noise parameters of $0.05 \mathrm{E}$ and $0.05 \mu \mathrm{Gal}$ are utilized for systematic noise of GGTs signals and GF respectively. Analyzing Fig. 3 and Fig. 4, and by considering all the three coordinates, the mean relative errors for object $\mathrm{R}$ are $0.5 \%$ along $x$-direction, $1.0 \%$ along $y$-direction and $3.1 \%$ along $z$-direction. Similarly, for object $\mathrm{S}$ these mean errors are

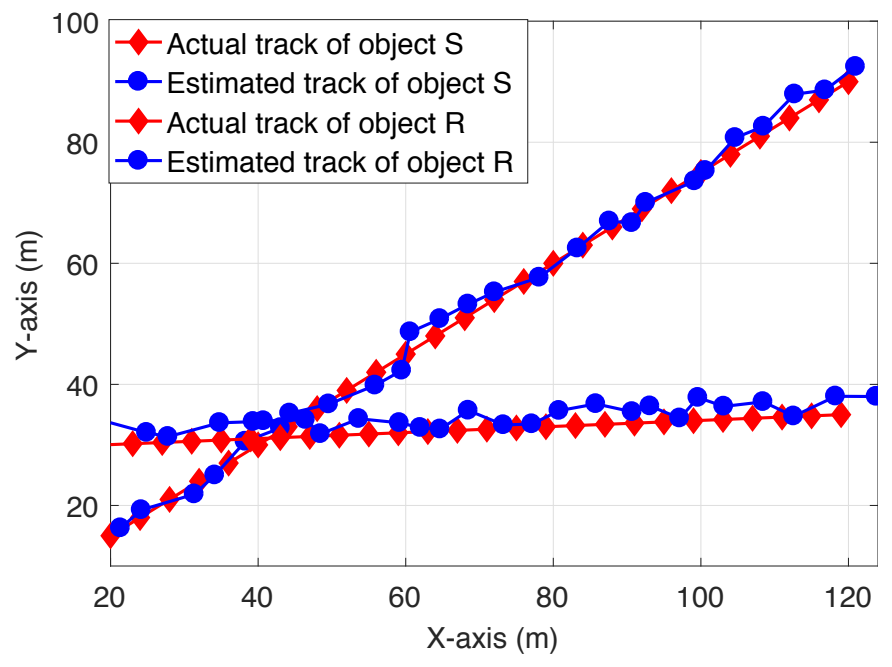

Fig. 4. Effect of noise on position estimation.

$1.9 \%$ along $x$-direction, $1.9 \%$ along $y$-direction and $1.9 \%$ along $z$-direction. By increasing the systematic noise level, the mean relative errors for both the objects gets increase. This increase for object $\mathrm{R}$ is now $1.3 \%$ along $x$-direction, $2.8 \%$ along $y$-direction and $7.5 \%$ along $z$-direction and for object $\mathrm{S}$ it is now $3.0 \%$ along $x$-direction, $4.9 \%$ along $y$-direction and $4.8 \%$ along $z$-direction.

Moreover, when this systematic noise level is made equal to the considered values as $0.05 \mathrm{E}$ for GGTs and $0.05 \mu \mathrm{Gal}$ for GF which is 2.5-times of instrumental precision value, the estimated positions again lies inside the cubic and prismatic objects. However, the absolute deviation for object $\mathrm{S}$ along $z$-direction gets increased from $2 m$ to $8 m$. But with same random noise of 2.5-times, the estimated positions for both the objects along $x-$ and $y$-directions are as follows; $6 \mathrm{~m}$ along $x$-direction and $6 \mathrm{~m}$ along $y$-direction for object $\mathrm{R}$ and $12 \mathrm{~m}$ along $x$-direction and $4 m$ along $y$-direction for object $\mathrm{S}$. The proposed work provides accurate estimation and reliable localization of two underwater objects for even greater level of noises. The point mass assumption is difficult if the separation between base station and mass bodies becomes greater than 3-times of the targeted dimensions [23]. This will lead to an increase in the localization error as well as the complexity for more than two object localization with same experimental setup.

Fig. 5 and Fig. 6 shows the convergence rate of the modified Levenberg-Marquardt algorithm compared to the traditional approach for the position estimation along the $x$ and $y$ coordinates respectively. It is clear from Fig. 5 and Fig. 6 that the proposed method converges in few iterations, i.e., 7 whereas the traditional algorithm converges at the 16th iteration.

\section{CONCLUSION}

This paper has proposed a novel technique for multiple object localization in an underwater wireless communication system using GF and GGTs signals. The technique utilizes 


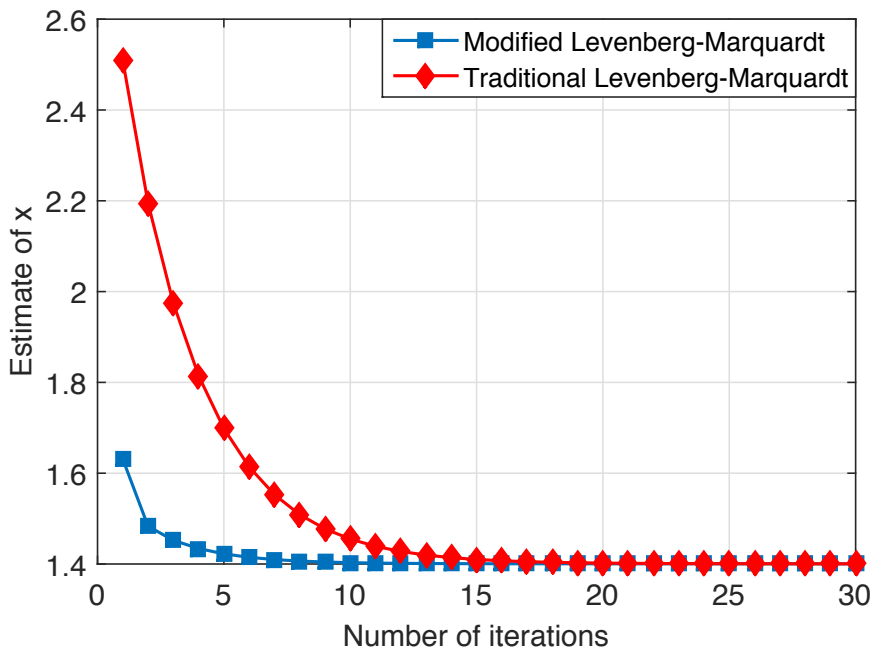

Fig. 5. Convergence rate for position estimation along $x$-axis.

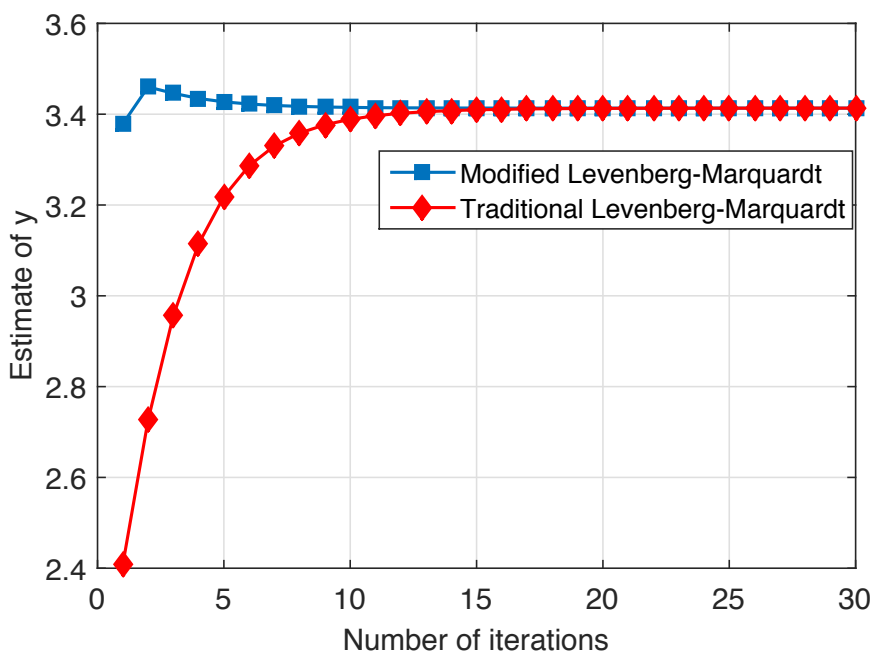

Fig. 6. Convergence rate for position estimation along $y$-axis.

few sets of regularized non-linear equations and parameters to find out the trajectories of various objects in an underwater environment. An algorithm has been developed using some synthetic data utilization that provides accurate information regarding the location of two underwater objects. Experimental results depict that this model provides efficient results not only in finding the complicated trajectories of the objects but also provide accurate measurements in the presence of high level noise. It can be concluded that the presented algorithm can work in wide scenarios and applications for localization of moving objects in underwater, ground and aerial environment.

\section{REFERENCES}

[1] N. Saeed, A. Celik, T. Y. Al-Naffouri, and M.-S. Alouini, "Underwater optical wireless communications, networking, and localization: A survey," arXiv preprint arXiv:1803.02442, 2018.
[2] H.-P. Tan, R. Diamant, W. K. Seah, and M. Waldmeyer, "A survey of techniques and challenges in underwater localization," Ocean Engineering, vol. 38, no. 14, pp. 1663 - 1676, 2011.

[3] N. Saeed, A. Celik, T. Y. Al-Naffouri, and M.-S. Alouini, "Underwater optical sensor networks localization with limited connectivity," in IEEE Int. Conf. on Acoustics, Speech and Signal Processing (ICASSP), Apr. 2018, pp. 1-5.

[4] J. Hariyono, V.-D. Hoang, and K.-H. Jo, "Moving object localization using optical flow for pedestrian detection from a moving vehicle," The Scientific World Journal, vol. 2014, 2014.

[5] W. K. Lyon, "Sonar, the submarine and the arctic ocean," The Journal of the Acoustical Society of America, vol. 32, no. 11, pp. $1513-1513$, 1960.

[6] D. J. Toal, C. Flanagan, W. B. Lyons, S. Nolan, and E. Lewis, "Proximal object and hazard detection for autonomous underwater vehicle with optical fibre sensors," Robotics and Autonomous Systems, vol. 53, no. 3 - 4, pp. 214 - 229, 2005.

[7] S. D. Gray, J. A. Parmentola, and R. LeSchack, "Estimating the weight of very heavy objects with a gravity gradiometer," of Physics D: Applied Physics, vol. 28, no. 11, p. 2378, 1995.

[8] Z. Yan, J. Ma, and J. Tian, "Accurate aerial object localization using gravity and gravity gradient anomaly," IEEE Geoscience and Remote Sensing Letters, vol. 12, no. 6, pp. 1214 - 1217, 2015.

[9] J. Tang, S. Hu, Z. Ren, C. Chen, X. Xiao, and C. Zhou, "Analytical formulas for underwater and aerial object localization by gravitational field and gravitational gradient tensor,' IEEE Geoscience and Remote Sensing Letters, vol. 14, no. 9, pp. 1557 - 1560, 2017.

[10] M. Beiki and L. B. Pedersen, "Eigenvector analysis of gravity gradient tensor to locate geologic bodies," Geophysics, vol. 75, no. 6, pp. $137-$ 149, 2010.

[11] Z. Ren, C. Chen, K. Pan, T. Kalscheuer, H. Maurer, and J. Tang, "Gravity anomalies of arbitrary $3 \mathrm{~d}$ polyhedral bodies with horizontal and vertical mass contrasts," Surveys in geophysics, vol. 38, no. 2, pp. $479-502$, 2017.

[12] Z. Ren, J. Tang, T. Kalscheuer, and H. Maurer, "Fast 3-d large-scale gravity and magnetic modeling using unstructured grids and an adaptive multilevel fast multipole method," Journal of Geophysical Research: Solid Earth, vol. 122, no. 1, pp. 79 - 109, 2017.

[13] L. Wu, X. Ke, H. Hsu, J. Fang, C. Xiong, and Y. Wang, "Joint gravity and gravity gradient inversion for subsurface object detection," IEEE Geoscience and Remote Sensing Letters, vol. 10, no. 4, pp. 865 - 869 2013.

[14] R. Castaldo, M. Fedi, and G. Florio, "Multiscale estimation of excess mass from gravity data," Geophysical Journal International, vol. 197, no. 3, pp. 1387 - 1398, 2014.

[15] W. Menke, Geophysical Data Analysis: Discrete Inverse Theory. Academic Press, 2012, vol. 45.

[16] A. Tikhonov and V. Y. Arsenin, Methods for solving ill-posed problems. John Wiley and Sons, Inc, 1977.

[17] R. Fletcher, "Modified marquaredt subroutine for nonlinear least square," Harwell Report AERE-R, vol. 6799, 1971.

[18] F. Bauer and M. A. Lukas, "Comparing parameter choice methods for regularization of ill-posed problems," Mathematics and Computers in Simulation, vol. 81, no. 1795 - 1841, 2011.

[19] L. Uieda and V. C. Barbosa, "Fast nonlinear gravity inversion in spherical coordinates with application to the south american moho," Geophysical Journal International, vol. 208, no. 1, pp. 162 - 176, 2016.

[20] M. V. Moody, H. J. Paik, and E. R. Canavan, "Three-axis superconducting gravity gradiometer for sensitive gravity experiments," Review of scientific instruments, vol. 73, no. 11, pp. 3957 - 3974, 2002.

[21] T. Hayes, K. Tiampo, J. Fernández, and J. Rundle, "A gravity gradient method for characterizing the post-seismic deformation field for a finite fault," Geophysical Journal International, vol. 173, no. 3, pp. 802 - 805, 2008.

[22] J. R. McKenna, H. Rim, and Y. Li, "Feasibility and limitations of void detection using gravity gradiometry," IEEE Transactions on Geoscience and Remote Sensing, vol. 54, no. 2, pp. 881 - 891, 2016.

[23] K.-M. Lee and M. Li, "Magnetic tensor sensor for gradient-based localization of ferrous object in geomagnetic field," IEEE Transactions on Magnetics, vol. 52, no. 8, pp. 1 - 10, 2006. 